\title{
Mémoire de source - présentation générale et revue des études dans la schizophrénie
}

\author{
A. FERCHIOU (1, 2, 3), F. SCHÜRHOFF (1, 2, 3), E. BULZACKA (1, 2, 3), \\ M. MAHBOULI (3), M. LEBOYER (1, 2, 3), A. SZÖKE (1, 2, 3)
}

(1) INSERM, U 955, Institut Mondor de Recherches Biomédicales (IMRB), département de Génomique Médicale, équipe de Psychiatrie Génétique, Créteil, F-94000, France;

(2) Université Paris-Est, Faculté de Médecine, IFR10, Créteil, F-94000, France;

(3) AP-HP, Hôpital Henri Mondor-Albert Chenevier, Pôle de Psychiatrie, Créteil, F-94000, France;

Correspondance :

Abdelaziz FERCHIOU

Groupe Hospitalo - Universitaire Mondor - Chenevier, pôle de psychiatrie

Hôpital Albert Chenevier, pavillon Hartman

40 rue de Mesly

94000 CRETEIL

Tél : + 33 (0)1 49813290

Email : aziz.ferchiou@inserm.fr 
Résumé : La mémoire de source constitue l'ensemble des processus cognitifs impliqués dans la reconnaissance de l'origine de l'information et participe de ce fait au fonctionnement adapté dans la vie quotidienne.

Cet article a pour objectif de faire une revue de la littérature des modèles théoriques et neuroanatomiques proposés pour expliquer la mémoire de source, des différentes méthodes d'évaluation utilisées, des facteurs (démographiques, cognitifs) qui influencent ce phénomène mnésique, ainsi que ses déficits dans différentes pathologies psychiatriques et en particulier la schizophrénie.

L'étude de la mémoire de source s'est montrée particulièrement pertinente pour l'étude de la schizophrénie en proposant des modèles explicatifs explorant les symptômes positifs, en particulier les hallucinations auditives. Toutefois, le déficit en mémoire de source n’est pas spécifique de la schizophrénie puisque des déficits ont été mis en évidence d'une part au cours d'autres pathologies psychiatriques et d'autre part en population générale en corrélation avec des traits schizotypiques.

La mémoire de source est un concept intéressant pour les recherches portant sur l'identification des substrats neuro - cognitifs des symptômes positifs. Toutefois, des améliorations méthodologiques, notamment une homogénéisation des paradigmes utilisés et une meilleure sélection des populations étudiées sont nécessaires.

Mots - clés : Mémoire de source, mémoire de réalité, schizophrénie, hallucinations auditives, symptômes positifs. 


\section{Source monitoring: general presentation and review of literature in schizophrenia}

\section{Summary:}

Source monitoring framework: Source monitoring refers to the ability to remember the origin of information. Three source monitoring processes can be distinguished: external source monitoring, internal or self-monitoring, and reality monitoring (i.e. discrimination between internal and external sources of information). Source monitoring decisions are based on memory characteristics recorded such as perceptions, contextual information or emotional reactions, and heuristic or more controlled judgement processes.

Brain structures: Several studies suggested that specific structures in the prefrontal and the mediotemporal lobes are the main areas implicated in source monitoring.

Assessment: A typical source monitoring paradigm includes an items generation stage, and a second stage of recognition of items (old versus new) and identification of their sources: external (usually the examiner) or internal (the subject). Several indices can be calculated based on the raw data such as the number of false alarms, attribution biases, or discrimination indexes. To date there is no standardized source monitoring task and differences in the type of items used (words, pictures), in the cognitive or emotional effort involved or in the delay between the two test stages, contribute to heterogeneity of results.

Factors influencing source monitoring: Factors like age (either very young or very old) and emotions influence source monitoring performances. Influence of gender was not properly explored, whereas the role of IQ and selective attention is still debated.

Source monitoring deficits in neurological disorders: Source monitoring deficits are observed mainly in disorders affecting fronto-temporal areas, like frontal trauma, Alzheimer's disease or frontotemporal dementia.

Source monitoring and schizophrenia: Source monitoring errors (e.g. external misattribution of selfgenerated information) are observed in schizophrenia, and seem to correlate with positive symptomatology, in particular auditory hallucinations, thought intrusion and alien control symptoms. These results are of particular interest in clinical research because source monitoring is one of the rare cognitive tests showing a correlation with the positive dimension. Source monitoring deficits have been proposed as a potential explanation for the positive symptoms and some, but not all studies lent support to this hypothesis. Heterogeneity of studied samples in particular different criteria to define hallucinating subjects (e.g. currently versus anytime during their lives) could explain the discordant results.

Source monitoring in psychiatric disorders from the schizophrenic spectrum: Source monitoring impairments were observed in pharmacological models of psychosis, in first degree relatives of schizophrenic patients, and also in general population associated with schizotypal dimensions. These 
results support a relationship between source monitoring deficits and some of the symptomatic dimensions of the schizophrenic spectrum but still await replication.

Source monitoring and other psychiatric disorders: Some studies found source monitoring deficits in other psychiatric conditions such as mania or obsessive - compulsive disorder. Thus, those studies suggest that source monitoring deficits may be not specific to schizophrenia.

Conclusion: Source monitoring competencies are critical for good (i.e. adapted) everyday functioning. Source monitoring deficits have been suggested as a potential explanation for some (or all) positive psychotic symptoms. However, to date methodological inconsistencies (especially with regard to tests design and choice of subjects’ samples) have precluded firm, definite conclusions.

Keywords: Source monitoring, reality monitoring, memory, schizophrenia, auditory hallucinations, positive symptoms. 


\section{Introduction}

La mémoire de source ou source monitoring représente l'ensemble des processus cognitifs impliqués dans la reconnaissance de l'origine de l'information. La source d'une information mémorisée est une donnée essentielle qui va donner plus ou moins de poids et de crédibilité à l’information. Des déficits en mémoire de source peuvent avoir des conséquences importantes sur la vie quotidienne y compris médico-légales. Ceci a été démontré par des études sur des phénomènes et situations tels que : le témoignage oculaire, la cryptomnésie (ou «plagiat par inadvertance » lorsque une personne reproduit une chose en étant convaincu d'être original), l'incorporation de la fiction (cinéma, télévision, journaux) dans les faits réels, les fausses mémoires autobiographiques, etc. [30]

Plusieurs études ont porté sur les mécanismes cognitifs normaux et pathologiques sous-tendant la mémoire de source et les conséquences de ses altérations dans des pathologies neurologiques ou psychiatriques.

Notre revue de la littérature est organisée en trois parties. Une première partie permettra de délimiter et définir les différentes composantes de la mémoire de source ainsi que les processus apparentés. La deuxième partie portera sur le fonctionnement normal des processus impliqués dans la mémoire de source et les facteurs qui l'influencent. Enfin la dernière partie résumera les données sur les altérations pathologiques, notamment dans la schizophrénie.

\section{Structure de la mémoire de source}

On distingue généralement trois sous-types de mémoire de source [30] :

- Le premier sous - type permet de différencier les informations générées intérieurement (pensées, éléments imaginaires) des informations provenant de l’extérieur (événements perçus). Ce mécanisme de discrimination interne - externe constitue la mémoire de réalité (reality monitoring).

- Le deuxième sous - type constitue la mémoire de source externe permet la discrimination entre différentes sources externes, par exemple entre deux personnes ou entre ce qui a été entendu dans la rue ou à la radio etc...

- Enfin, le troisième sous - type constitue la mémoire de source interne (parfois appelée self monitoring) permettant de distinguer les différents processus par lesquels un sujet a généré une information: par exemple, différencier ce que le sujet a pensé de ce qu'il a dit.

Lors de l'élaboration du souvenir (processus d'encodage), les différentes modalités de l'information (ou signaux) sont enregistrées. Ces signaux comportent les caractéristiques perceptives (sons, couleurs, etc...), le contexte temporo-spatial, les réactions émotionnelles et les opérations cognitives engagées (élaboration, organisation). Ces caractéristiques varient en fonction de la source et contribuent par la suite à son identification. Par exemple, par rapport à la mémoire d'événements imaginés, la mémoire d'événements externes a tendance à inclure davantage d’informations 
perceptuelles, temporo-spatiales, sémantiques et affectives et moins d'informations concernant les opérations cognitives.

En conséquence, un souvenir comportant beaucoup de détails visuels et spatiaux mais impliquant peu d'opérations cognitives sera considéré comme d'origine externe (mécanisme entrant en jeu dans la mémoire de réalité).

Plusieurs «décisions » concernant la mémoire de source sont faites ainsi rapidement sur la base des caractéristiques qualitatives du souvenir.

Parfois, ces caractéristiques ne suffisent pas à identifier avec certitude l'origine d'une information. Dans ce cas, des processus stratégiques, plus lents, faisant appel au raisonnement, au jugement (« est ce que ceci est plausible ? ») entrent en jeu. On différencie, donc, un processus de décision habituellement utilisé, rapide, dit «automatique » ou "heuristique » et un deuxième processus de jugement entrant en jeu seulement en cas d'échec du premier, dit «contrôlé » ou «systématique » [30].

\section{Phénomènes apparentés à la mémoire de source}

Dans la psychologie expérimentale, la reconnaissance ancien - nouveau représente le processus cognitif permettant de différencier entre un item auparavant présenté, dit ancien et un item jamais présenté, dit nouveau.

En pratique, il est difficile de distinguer la reconnaissance ancien - nouveau du sentiment de familiarité car, les nouveaux items présentés (comme les anciens) ne sont pas totalement inconnus au sujet.

Les phénomènes de reconnaissance et d'identification de la source font appel à des processus stratégiques différents. La reconnaissance ancien - nouveau, ou la familiarité, constituent une évaluation globale et indifférenciée. Ces processus nécessitent une information moins complète et moins différenciée que la mémoire de source et précèdent dans le temps l'identification plus spécifique de la source. (Pour une discussion plus détaillée voir Johnson et al. [30])

\section{Structures cérébrales impliquées dans la mémoire de source}

La mémoire de source étant intimement liée au concept même de mémoire épisodique, il parait évident que les structures cérébrales impliquées dans ce phénomène englobent les régions habituellement associées aux processus d'encodage, de stockage et de récupération d'une trace mnésique, notamment les régions préfrontales et médio - temporales [14]. Toutefois, certaines particularités semblent exister. Ainsi, Davachi et al. [16] ont mis en évidence une activation spécifique de l'hippocampe et du cortex parahippocampique postérieur dans la mémorisation de la source, alors que la mémoire de l'item corrélait uniquement avec l'activation du cortex périrhinal, démontrant ainsi l'existence de deux processus distincts participant à la formation d'une trace mnésique complète. Cansino et al. [14] ont identifié les structures impliquées de manière différentielle lors de l'encodage et la récupération de 
l'origine de l'item. En effet, les régions latéro - occipitales droites et le cortex préfrontal gauche augmenteraient leur activité lors du processus d'encodage. En revanche, la formation hippocampique droite et le cortex préfrontal gauche seraient dédiés à la récupération.

Les études en neuroimagerie fonctionnelle ont par ailleurs mis en évidence une implication du cortex préfrontal (CPF), notamment l'aire 10 de Broadman, dans d'encodage et la récupération de l'information contextuelle (pour revue, voir Turner et al. [45]). Il semble que le CPF rostro -latéral joue un rôle non spécifique dans la récupération de la source, alors que la partie rostro - médiane serait dédiée à la récupération des détails contextuels concernant l’information générée par le sujet.

De plus, le CPF médian ventro - caudal serait mis en jeu lorsque le sujet doit déterminer si les stimuli imaginés ont été uniquement produits par lui-même, ou s’ils avaient été antérieurement perçus [45].

Si le déficit en mémoire de source semble être relié à un déficit d'activité d'une ou de plusieurs de ces régions cérébrales, d'autres auteurs mettent en cause un défaut de connectivité entre ces régions via un déficit de copie d'efférence [20].

\section{Evaluation de la mémoire de source}

Le format classique d'un test de mémoire de source comprend deux parties : une première partie de "production» des items (informations) et une deuxième de reconnaissance (test de la mémoire de source à proprement parler). La première partie implique la production ou la présentation des items provenant de différentes sources: externes (une ou plusieurs sources externes; items visuels ou auditifs, etc...) ou internes (items lus ou imaginés par le sujet lui-même). Dans la deuxième partie, ces items (anciens) sont présentés de manière aléatoire au sujet, mélangés (éventuellement) à d'autres items (nouveaux). On demande au sujet d'indiquer pour chaque item s'il est ancien ou nouveau et, pour les items «anciens », de préciser leur origine (lu ou généré par lui-même, généré par l'expérimentateur, etc...) [6,21,26,46].

Ainsi, à partir de là, plusieurs indices peuvent être calculés, dont les plus utilisés sont [15] :

1. le nombre total (ou le pourcentage) de réponses correctes.

2. le "hit rate " c'est à dire le nombre (ou le pourcentage) d'items correctement reconnus comme anciens, et ce indépendamment de la reconnaissance correcte ou non de leur origine.

3. les fausses alarmes qui sont les items nouveaux que le sujet désigne, de manière erronée, comme déjà présentés (anciens).

4. les biais d'attribution (ou fausses attributions) à une source (le nombre d'items attribués à tort à une source donnée).

5. l'index de discrimination d'une source donnée : le nombre de fois qu'une source donnée a été correctement reconnue comme origine de l'information rapporté au nombre total d'items correctement reconnus comme anciens.

Il existe une grande variabilité des tests de mémoire de source selon les consignes données au sujet, le délai entre la présentation des items et la phase de reconnaissance et la difficulté de la tâche. En ce qui 
concerne les consignes, certains auteurs, en essayant de se rapprocher au maximum des conditions " écologiques », ne prévoient pas d'informer le sujet sur le fait qu'il devra se souvenir des items et de leur source $[6,46]$, alors que dans d'autres études le sujet reçoit cette instruction [32,39].

L'intervalle entre les deux parties du test peut également varier d'un test à un autre : rappel immédiat [9,32,36] ou différé $[4,21,26]$. Toujours dans cette tentative de se rapprocher des conditions « écologiques », certaines tâches proposent non seulement un intervalle de temps entre production et rappel, mais intercalent d'autres tâches cognitives entre les deux phases du test ayant pour but d'empêcher le maintien des informations dans la mémoire de travail [35,39,46].

D’autres auteurs ont proposé des tâches de difficulté variable: certaines catégories présentées contiennent des mots de la vie quotidienne (exemple : fruits) et demandent peu d'efforts cognitifs ou émotionnels alors que d'autres peuvent demander plus d'efforts sur le plan cognitif (exemple : pays d’Europe pour des sujets non européens) ou émotionnel (exemple : « les sentiments les plus intenses que vous avez ressentis ») [39].

\section{Variables qui influencent la mémoire de source}

Quelques études ont essayé d'identifier les facteurs qui, en dehors des situations pathologiques (troubles neurologiques ou psychiatriques), influencent l'efficacité de la mémoire de source. Parmi ces variables, on compte les facteurs démographiques, l'état affectif du sujet et les facteurs cognitifs.

Des résultats plus faibles aux tests évaluant la mémoire de source ont été mis en évidence aux deux extrémités de la vie, chez les enfants et chez les sujets âgés. Alors que chez l'enfant le déficit parait sélectif, portant davantage sur la mémoire de source interne [19], chez le sujet âgé il est plus généralisé [18,43]. A notre connaissance, aucune étude ne s'est intéressée à l'influence du sexe sur les performances à la mémoire de source.

Contrairement aux caractéristiques perceptuelles, contextuelles et sémantiques, les émotions ne sont pas un bon signal discriminatif pour l'origine d'une information. De plus, leur présence peut, parfois, perturber l'encodage et le rappel de l'origine de l'information. Par exemple, des scores élevés d'anxiété ou de dépression au moment de l'encodage ou du rappel ont été associés à des déficits en mémoire de source [31].

La mémoire de source dépend de la qualité de l'encodage initial de l'information. Tous les éléments perturbant ce processus (par exemple : stress, éléments distracteurs, alcool...) rendent l'encodage de signaux potentiellement pertinents moins efficace. Ces éléments peuvent également perturber les processus décisionnels et de jugement et, par conséquence, augmenter le risque d'erreurs dans l'identification de la source [30].

Il a été démontré que l'augmentation des demandes attentionnelles (tâche d'attention divisée) dans la phase de reconnaissance perturbait les processus de jugement contrôlés, alors que ceux heuristiques n’étaient pas atteints [17]. 
Quant au QI, si plusieurs études se sont intéressées à son éventuelle influence sur la mémoire de source dans des situations pathologiques telles que la schizophrénie (cf infra), aucune n’a été réalisée chez des sujets sains.

\section{Altérations pathologiques de la mémoire de source}

\section{Pathologies neurologiques}

Toute pathologie affectant les régions médio - temporales et surtout frontales peut altérer la mémoire de source. En effet, il semble que les sujets souffrant de traumatismes frontaux [13, 43], de maladie d'Alzheimer [13] ou de démence fronto - temporale [40] présentent plus d'erreurs de mémoire de source que les sujets contrôles, notamment plus de fausses alarmes. Ce déficit en mémoire de source serait à l'origine de symptômes de type « fausses reconnaissances ».

\section{Pathologies psychiatriques}

La plupart des études portant sur la mémoire de source réalisées chez des sujets souffrant de pathologies psychiatriques concernent la schizophrénie. En effet, il existe peu d'études qui se soient intéressées à la mémoire de source dans les troubles psychiatriques autres que la schizophrénie ; celles - ci seront présentées à la fin de cette section.

\section{La schizophrénie}

\section{Etudes comparant les schizophrènes aux sujets sains}

La plupart des études s'accordent sur le fait que les schizophrènes ont plus de difficultés dans la reconnaissance de l'origine de l'information que les témoins sains d'une manière générale et quel que soit le type de mémoire de source étudiée [6,21,26,32,46,47]. Ce constat a rapidement posé le problème de l'existence de facteurs de confusion tels que l'influence du traitement pharmacologique sur les performances cognitives par exemple, ou l'influence d'autres variables comme l'attention sélective ou le QI.

Les psychotropes ne semblent pas expliquer les mauvaises performances des schizophrènes en mémoire de source puisque des déficits à ce niveau sont retrouvés chez des patients naïfs de tout traitement [32]. Bien au contraire, les antipsychotiques pourraient améliorer ces mauvaises performances [33].

Concernant l'attention sélective, deux études ont suggéré son implication dans les performances à la mémoire de source chez les sujets schizophrènes. Le déficit en attention sélective chez ces derniers entraînerait une incapacité à inhiber les stimuli non pertinents lors de la phase d'encodage ou de stockage de l'information. Mais ces études ont utilisé des paradigmes différents sur des échantillons relativement réduits $[5,26]$. Notre équipe n’a pas répliqué ce résultat sur un échantillon plus important (article en préparation). 
De la même manière, pour le QI dont il est admis qu’il est généralement plus bas chez les patients schizophrènes, son éventuelle influence sur les performances à la mémoire de source reste controversée, avec des études positives [36,38,46] et d’autres négatives [41].

La question de la spécificité du déficit en mémoire de source dans la schizophrénie (les patients schizophrènes étant moins performants que les témoins dans la quasi-totalité des tests neurocognitifs) et l'intérêt d'étudier ce phénomène dans cette pathologie ont également été abordés.

Ces réflexions ont amené à rechercher au sein de la schizophrénie des corrélations plus spécifiques entre mémoire de source et certains symptômes ou dimensions symptomatiques.

Les différentes études se sont intéressées respectivement 1) aux hallucinations auditives de manière spécifique, 2) aux symptômes positifs de manière générale, ou 3) aux différentes dimensions symptomatiques de la schizophrénie

\section{Mémoire de source et symptômes schizophréniques}

1) Les hallucinations

Plusieurs études ont montré que les schizophrènes qui présentent des hallucinations auditives ont plus fréquemment tendance à produire des fausses alarmes lors de la phase de reconnaissance ancien nouveau item, par rapport aux sujets schizophrènes qui ne présentent pas d'hallucinations [6], ainsi qu'une tendance plus importante aux fausses attributions des items générés par eux-mêmes à une source externe $[4,6,9,47]$. Certains travaux ont renforcé cette relation spécifique entre hallucinations auditives et déficit en mémoire de source en montrant une amélioration des performances à la mémoire de source conjointement à la réduction des hallucinations par stimulation magnétique transcrânienne, sans qu'il n’y ait d'amélioration des autres symptômes positifs [10].

A partir de ces données ainsi que des données expérimentales, cliniques, éléctro-physiologiques et de neuro-imagerie fonctionnelle reliant les phénomènes hallucinatoires auditifs au langage notamment au discours intérieur [24,29], plusieurs auteurs ont suggéré que les déficits en mémoire de source pourraient expliquer la survenue des hallucinations.

Si les modèles proposés s'accordent sur le fait que le sujet halluciné attribue ses propres productions à une source externe, ils diffèrent selon la nature hypothétique des mécanismes responsables de cette mauvaise attribution.

Selon Frith [22], un trouble central et stable du monitoring des actions et des intentions perturberait l’identification de leur origine. En conséquence, le discours intérieur généré par le sujet n’est pas reconnu comme initié par lui-même. Cette absence d’information conduit le sujet à ne pas se reconnaître comme auteur de ce discours, qui ne peut être alors qu'étranger et perçu comme des « voix » extérieures.

Ce modèle basé sur un trouble du self monitoring est renforcé par les travaux de Franck et al. [21] dont l'hypothèse postule que les hallucinations résultent de l'incapacité de discriminer entre le discours intérieur et le discours verbalisé. 
Contrairement à ce modèle qui suggère que l'information permettant de reconnaître la source n’a pas été encodée, ceux proposés par Bentall [3,4] ou Morrison [2,35] suggèrent que les hallucinations seraient le résultat d'un trouble au niveau des processus impliqués dans la discrimination entre les événements réels et imaginaires (mémoire de réalité). Ces processus seraient biaisés lors de la phase de récupération de l'information par des facteurs cognitifs (par exemple attribuer à l'expérimentateur les items demandant un effort cognitif important) [4] ou affectifs tels que la valence émotionnelle de l'information [35] ou l'état thymique du sujet [2]. Par pur mécanisme «d'auto défense », le sujet schizophrène peut ainsi attribuer à l'extérieur des pensées jugées trop hostiles, angoissantes ou intolérables [3].

Il est évident que les deux modèles ne sont pas mutuellement exclusifs et que la survenue d'hallucinations pourrait combiner une incapacité du sujet à s'identifier comme auteur de la pensée ou de l'action et une tendance à attribuer à l'extérieur ses propres expériences.

Toutefois, cette relation spécifique entre mémoire de source et hallucinations n’a pas été systématiquement retrouvée [39,46].

L’hétérogénéité des résultats est en grande partie en rapport avec une hétérogénéité de la définition des groupes de sujets hallucinés versus non hallucinés, et dépend de manière plus générale de la considération des hallucinations comme un symptôme - trait ou état [39].

Ainsi, selon les études, les patients hallucinés présentent au moment même du test des hallucinations [4], ont eu des hallucinations dans la semaine précédant le test [44] ou quotidiennement depuis 2 mois [9].

Parallèlement, le groupe de patients non hallucinés peut être formé aussi bien de patients qui n’ont jamais halluciné [4] que de patients qui ne présentent plus d’hallucinations depuis un temps donné [9]. Un autre problème qui complique l'interprétation des résultats est que les patients non hallucinés ont probablement une pathologie moins sévère ou une meilleure réponse au traitement que le groupe de sujets hallucinés [39].

L’hétérogénéité des résultats a suggéré que la corrélation entre hallucinations et déficits en mémoire de source n’est peut être pas spécifique.

\section{2) La symptomatologie positive}

Ce sont les travaux de Frith [22,23] qui ont suggéré que le trouble du monitoring interne pourrait expliquer la survenue non seulement des hallucinations mais également d'autres symptômes schizophréniques notamment le délire d'influence ou les pensées imposées, et ce par le même trouble du self monitoring qui empêche le schizophrène de se reconnaître comme auteur de ses pensées ou de ses actes.

Cette hypothèse a été reprise par le concept « d'agnosie autonœtique » de Keefe [32], c'est - à - dire «l’incapacité d’identifier les événements mentaux générés par soi » [32], puis par les résultats de l'étude de Stirling et al. [41]. 
L'étude de Keefe et al. [32] est particulièrement intéressante car elle est la seule à avoir évalué chez les patients schizophrènes le déficit dans les 3 types de mémoire de source. C’est uniquement au test de mémoire de source interne que les schizophrènes avec «symptômes cibles » (pensées, émotions et actes imposés, commentaire des actes) ont significativement plus de difficulté que le reste des sujets schizophrènes.

Dans l'étude de Brébion et al. [6], des erreurs de mémoire de réalité sont constatées aussi bien chez les sujets délirants sans hallucinations que chez les sujets délirants avec hallucinations, mais de façon plus importante chez ces derniers. Les auteurs concluent que le délire et les hallucinations pourraient être corrélés avec des altérations des processus de mémoire de source à 2 niveaux différents : les sujets hallucinés étant souvent en plus délirants, ils sont donc plus déficients.

En comparant des sujets schizophrènes hallucinés versus non - hallucinés au cours d'un test de mémoire de réalité et un test de mémoire de source interne, Brunelin et al. [9], trouvent des différences entre les 2 groupes uniquement pour la performance en mémoire de réalité, les sujets présentant des hallucinations étant significativement moins performants. Les auteurs émettent l'hypothèse que la déficience au test de mémoire de réalité pourrait être un marqueur-état des hallucinations acousticoverbales alors que la déficience au test de mémoire de source interne serait un marqueur-trait de la schizophrénie en général, en rapport avec plusieurs symptômes positifs.

Concernant les études dimensionnelles, la corrélation entre dimension positive et schizophrénie a été décrite [7,42] mais n’a pas été confirmée par toutes les études [44,46].

3) Autres symptômes

Très peu d'études se sont intéressées aux autres dimensions symptomatiques de la schizophrénie.

Seules deux études ont retrouvé une corrélation entre mémoire de source et troubles du cours de la pensée [25,36], résultat non retrouvé par Brébion et al. [6].

Quant à la symptomatologie négative, les résultats sont controversés avec des corrélations positives $[44,46]$, ou négatives $[7,42]$ entre ces symptômes et les performances aux tests de mémoire de source.

\section{Mémoire de source et phénomènes apparentés aux symptômes schizophréniques}

Les résultats chez les patients schizophrènes sont souvent difficiles à interpréter à cause de l'existence de nombreux facteurs de confusion (par exemple médication, hospitalisations, etc...). Pour cette raison des études ont été réalisées chez des sujets présentant des phénomènes apparentés à la schizophrénie : modèles pharmacologiques de psychose, trouble schizotypique, etc...

La kétamine, substance anesthésique et hallucinogène, antagoniste du récepteur N-Méthyl-D-Aspartate (NMDA), est souvent utilisée chez le sujet sain comme un modèle pharmacologique de la schizophrénie. Des volontaires sains sous kétamine présentent une altération de la mémoire de réalité même à des doses n’induisant pas cliniquement de symptômes psychotiques. Toutefois, contrairement 
à ce qui a été décrit dans la schizophrénie, les sujets sous kétamine auraient plutôt tendance à un biais d'attribution interne [28]. Ce résultat remet en question la pertinence de ce modèle de la schizophrénie. D’autres études menées en population non malade ont retrouvé une corrélation entre faibles performances à la mémoire de source et respectivement propension à halluciner [34], propension à délirer [1] et scores élevés de schizotypie [37].

Brunelin et al. [11] en comparant lors d'une tâche de mémoire de réalité des patients schizophrènes, leurs apparentés sains et des sujets contrôles, trouvent que les performances des apparentés se situent à un niveau intermédiaire par rapport aux deux autres groupes. Ils suggèrent que le déficit en mémoire de source peut être considéré comme un marqueur de vulnérabilité à la schizophrénie. Toutefois, notre équipe n’a pas répliqué ce résultat [44].

Au total, les résultats chez les sujets non psychotiques confortent l'idée d'une relation entre déficit en mémoire de source et certaines dimensions symptomatiques du spectre de la schizophrénie en éliminant l'effet des facteurs de confusion liés à la maladie et sa prise en charge. Par contre, il est difficile de conclure si le déficit en mémoire de source est spécifique de la schizophrénie ou simplement en rapport avec certains symptômes ou dimensions symptomatiques non exclusivement propres à la schizophrénie.

\section{Autres pathologies psychiatriques}

Peu d'études se sont intéressées à l'évaluation de la mémoire de source dans d'autres pathologies psychiatriques, ce qui ne permet pas de conclure à l'existence de déficits de mémoire de source dans ces pathologies.

Parmi ces études citons celle de Harvey [25] qui a retrouvé une corrélation entre troubles du cours de la pensée et déficit en mémoire de source externe chez des patients maniaques, ce qui suggère que le déficit en mémoire de source est plus lié à des symptômes spécifiques qu’à un diagnostic.

Quelques études se sont intéressées aux performances à la mémoire de réalité chez les patients souffrant de Trouble Obsessionnel et Compulsif (TOC), l'hypothèse étant que les patients souffrant de TOC, notamment les vérificateurs, auraient tendance à confondre les mémoires des actions exécutées et celles des actions imaginées. Mais les résultats sont contradictoires. Ainsi, certaines études ont décrit une corrélation positive [38] ou négative [8] entre degré de gravité du TOC et mémoire de réalité, alors que d'autres études ne retrouvent pas de corrélation [27].

Enfin, une étude menée par Brunelin et al. (2008) ne retrouve pas de déficit en mémoire de source chez des sujets hospitalisés présentant un épisode dépressif majeur [12].

\section{Conclusion}

La mémoire de source est un processus cognitif régulièrement sollicité, nécessaire pour la prise de toute décision et essentiel pour un fonctionnement adapté. Malgré son importance ce processus a été peu exploré à ce jour ce qui explique les nombreuses questions qui subsistent. 
Dans le domaine de la psychiatrie, la mémoire de source a été essentiellement étudiée dans la schizophrénie. Les résultats obtenus ont conduit à des modèles explicatifs de l'ensemble ou d'une partie de la symptomatologie schizophrénique.

L’hétérogénéité des résultats suggère la nécessité d’une standardisation des épreuves et une meilleure définition des groupes de patients.

Les tests de mémoire de source ont un intérêt particulier dans l'étude des dimensions psychotiques puisqu'ils sont parmi les rares tests cognitifs à avoir montré, de manière répétée, une corrélation négative avec la symptomatologie positive. Ce résultat "atypique » pour un test cognitif pourrait représenter un fort argument pour la spécificité du déficit de la mémoire de source.

Enfin, l'étude de la mémoire de source pourrait avoir un intérêt dans l'évaluation de la pertinence de certains modèles expérimentaux de la schizophrénie ou dans l'évaluation de l'efficacité de certaines thérapeutiques proposées dans cette pathologie en offrant une approche plus fine et plus objective que celle de la clinique.

\section{Références}

[1] Allen P, Freeman D, Johns L, et al. Misattribution of self-generated speech in relation to hallucinatory proneness and delusional ideation in healthy volunteers. Schizopr Res 2006;84:281-8.

[2] Baker CA, Morrison AP. Cognitive processes in auditory hallucinations: attributional biases and metacognition. Psychol Med 1998;28:1199-208.

[3] Bentall RP. The illusion of reality: a review and integration of psychological research on hallucinations. Psychol Bull 1990;107:82-95.

[4] Bentall RP, Baker GA, Havers S. Reality monitoring and psychotic hallucinations. Br J Clin Psychol 1991;30:213-22.

[5] Brébion G, Smith MJ, Gorman JM, et al. Reality monitoring failure in schizophrenia: the role of selective attention. Schizophrenia Res 1996;22:173-80.

[6] Brébion G, Amador X, David A, et al. Positive symptomatology and source-monitoring failure in schizophrenia - an analysis of symptom-specific effects. Psychiatry Res 2000;95:119-31.

[7] Brébion G, Gorman JM, Amador X, et al. Source monitoring impairments in schizophrenia: characterisation and associations with positive and negative symptomatology. Psychiatry Res 2002;112:27-39.

[8] Brown HD, Kosslyn SM, Breiter HC, et al. Can patients with obsessive-compulsive disorder discriminate between percept and mental images? A signal detection analysis. J Abnorm Psychology 1994;103:445-54. 
[9] Brunelin J, Combris M, Poulet E, et al. Source monitoring deficits in hallucinating compared to non-hallucinating patients with schizophrenia. European Psychiatry 2006;21:259-61.

[10] Brunelin J, Poulet E, Bediou B, et al. Low frequency repetitive transcranial magnetic stimulation improves source monitoring deficit in hallucinating patients with schizophrenia. Schizophr Res 2006;81:41-5.

[11] Brunelin J, D’Amato T, Brun P, et al. Impaired verbal source monitoring in schizophrenia : An intermediate trait vulnerability marker ? Schizophr Res 2007;89:287-92.

[12] Brunelin J, Poulet E, Marsella S, et al. Selective source monitoring impairement in patients with schizophrenia compared to healthy and major depressive disorder subjects. European review of applied psychology 2008;58:105-10.

[13] Budson AE, Sullivan AL, Mayer E, et al. Suppression of false recognition in Alzheimer's disease and in patients with frontal lobe lesions. Brain 2002;125:2750-65.

[14] Cansino S, Maquet P, Dolan RJ, et al. Brain activity underlying encoding and retrieval of source memory. Cereb Cortex 2002;12:1048-56.

[15] Corwin J. On measuring discrimination and response bias: unequal numbers of targets and distractors and two classes of distractors. Neuropsychology 1994;8:110-17.

[16] Davachi L, Mitchell JP, Wagner AD. Multiple routes to memory: Distinct medial temporal processes build item and source memories. Proc Natl Acad Sci 2003;100:2157-61.

[17] Dywan J, Segalowitz SJ, Webster L. Source monitoring: ERP evidence for greater reactivity to nontarget information in older adults. Brain Cogn 1998;36:390-430.

[18] Dywan J, Segalowitz S, Arsenault A. Electrophysiological response during source memory decisions in older and younger adults. Brain Cogn 2002;49:322-40.

[19] Foley MA, Johnson MK. Confusion between memories for performed and imagined actions: a developmental comparision. Child Dev 1985;56:1145-55.

[20] Ford JM, Mathalon DH. Corollary discharge dysfunction in schizophrenia: Can it explain auditory hallucinations? Int J Psychophysiol 2005;58:179-89.

[21] Franck N, Rouby P, Daprati E, et al. Confusion between silent and overt reading in schizophrenia. Schizophr Res 2000;41:357-64.

[22] Frith CD. The positive and negative symptoms of schizophrenia reflect impairments in the perception and initiation of action. Psychol med 1987;17:631:48.

[23] Frith CD, Done DJ. Experiences of alien control in schizophrenia reflect a disorder in the central monitoring of action. Psychol Med 1989;19:359-63.

[24] Gould LN. Auditory hallucinations and subvocal speech; objective study in a case of schizophrenia. J Nerv Ment Dis 1949;109:418-27.

[25] Harvey PD. Reality monitoring in mania and schizophrenia. J Nerv Ment Dis 1985;173:67-73.

[26] Henquet C, Krabbendam L, Dautzenberg J, et al. Confusing thoughts and speech: source monitoring and psychosis. Psychiatry Res 2005;133:57-63. 
[27] Hermans D, Martens K, De Cort K, et al. Reality monitoring and metacognitive beliefs related to cognitive confidence in obsessive-compulsive disorder. Behav res ther 2003;41:383-401.

[28] Honey GD, O’Loughlin C, Turner DC, et al. The effects of a subpsychotic dose of ketamine on recognition and source memory for agency: implications for pharmacological modelling of core symptoms of schizophrenia. Neuropsychopharmacology 2006;31:413-23.

[29] Inouye T, Shimizu A. The electromyographic study of verbal hallucination.J Nerv Ment Dis 1970;151:415-22.

[30] Johnson MK, Hashtroudi S, Lindsay DS. Source Monitoring. Psychol Bull 1993;114:3-28.

[31] Johnson MK. Memory and reality. Am Psychol 2006;61:757-71.

[32] Keefe RSE, Arnold MC, Bayen UJ, et al. Source monitoring deficits in patients with schizophrenia; a multinomial modelling analysis. Psychol Med 1999;29:903-14.

[33] Keefe RSE, Poe MP, McEvoy JP, et al. Source monitoring improvement in patients with schizophrenia receiving antipsychotic medications. Psychopharmacology (Berl) 2003;169:3839.

[34] Larøi F, Van der Linden M, Marczewski P. The effects of emotional salience, cognitive effort and meta-cognitive beliefs on a reality monitoring task in hallucination-prone subjects. $\mathrm{Br} \mathrm{J}$ Clin Psychol 2004;43:221-33.

[35] Morrison AP, Haddock G. Cognitive factors in source monitoring and auditory hallucinations. Psychol Med 1997;27:669-79.

[36] Nienow TM, Docherty NM. Internal source monitoring and thought disorder in schizophrenia. J Nerv Ment Dis 2004;192(10):696-700.

[37] Peters MJV, Smeets T, Giesbrecht T, et al. Confusing action and imagination. Action source monitoring in individuals with schizotypal traits. J Nerv Ment Dis 2007;195:752-7.

[38] Rubenstein CS, Scheffler C, Peynircioglu ZF, et al. Memory in sub-clinical obsessivecompulsive checkers. Behav Res Ther 1993;31:759-65.

[39] Seal ML, Crowe SF, Cheung P. Deficits in source monitoring in subjects with auditory hallucinations may be due to differences in verbal intelligence and verbal memory. Cognit Neuropsychiatry 1997;2:273-90.

[40] Simons JS, Verfaellie M, Galton CJ, et al. Recollection-based memory in frontotemporal dementia: implications for theories of long-term memory. Brain 2002;125:2523-36.

[41] Stirling JD, Hellewell JS, Quraishi N. Self-monitoring dysfunction and the schizophrenic symptoms of alien control. Psychol Med 1998;28:675-83.

[42] Stirling JD, Hellewell JSE, Ndlovu D. Self-monitoring dysfunction and the positive symptoms of schizophrenia. Psychopathology 2001;34:198-202.

[43] Swick D, Senkfor AJ, Van Petten C. Source memory retrieval is affected by aging and prefrontal lesions: Behavioural and ERP evidence. Brain Research 2006;1107:161-76. 
[44] Szöke A, Méary A, Ferchiou A, et al. Correlations between cognitive performances and psychotic or schizotypal dimensions. Eur Psychiatry 2009;24:244-50.

[45] Turner MS, Simons JS, Gilbert SJ, et al. Distinct roles for lateral and medial rostral prefrontal cortex in source monitoring of perceived and imagined events. Neuropsychologia 2008;46:144253.

[46] Vinogradov S, Willis-Shore J, Poole JH, et al. Clinical and neurocognitive aspects of source monitoring errors in schizophrenia. Am J Psychiatry 1997;154:1530-7.

[47] Woodward TS, Menon M, Withman JC. Source monitoring biases and auditory hallucinations. Cognit Neuropsychiatry 2007;12:477-94. 